\title{
Should preterm labour and preterm premature rupture of membranes be treated with antibiotics?
}

\author{
AP Macdonald \\ Department of Obstetrics and Gynaecology, University of Pretoria, Pretoria, South Africa
}

\section{Introduction}

Preterm Labour (PTL) and Preterm Premature Rupture of Membranes (PPROM), both defined as occurring spontaneously before 37 completed weeks of pregnancy, have long been recognized as the major contributor to perinatal mortality and morbidity. The outcome for the baby deteriorates proportionately the earlier the incident occurs. It is estimated that spontaneous preterm birth is responsible for $21.8 \%$ of perinatal deaths or 6346 babies per year in South Africa. ${ }^{1}$ Preterm birth is less common in first world countries $\left( \pm 12 \%\right.$ of deliveries $\left.{ }^{2}\right)$ but in spite of intensive research the figures continue to increase. A disproportionate amount of the costs incurred in managing neonates are caused by preterm delivery. Approximately 30\% of preterm deliveries are indicated - the most common reasons being pre-eclampsia, fetal distress, intra-uterine growth restriction and obstetric haemorrhage. ${ }^{3}$ This category continues to increase because of the increased in multiple births from assisted reproductive treatment, changes in obstetric management between 34-37 weeks gestation and induction of labour in very preterm infants coupled with the willingness to resuscitate extremely low birth weight infants. Of the remaining two thirds of preterm deliveries approximately 60\% are associated with infection and this group is divided equally between PTL and PPROM. ${ }^{4}$

For many years PTL and PPROM were considered overlapping causes in the spectrum of preterm delivery and therefore the same management of tocolysis (if contractions were present), steroids and antibiotics were prescribed. The first aim was to delay labour for 24 hours to "get steroids on board" and thereafter any prolongation of the pregnancy was a bonus. In spite of an enormous volume of research, the causes of PTL and PPROM remain elusive and at present there is no effective prevention and only limited success with treatment.

\section{Aetiology of PTL and PPROM}

Infection is associated with PTL and PPROM and is generally considered to be an ascending infection from the lower genital tract. ${ }^{5}$ Most of the bacteria of the vagina can be readily identified but this does not necessarily imply upper genital tract infection as not all organisms will cause infection and the size of the innoculum and virulence of the organism needed to cause infection has never been determined. Organisms in the vagina are readily accessible but to isolate organisms from the upper genital tract (decidua, membranes, amniotic fluid and fetus) is apart from performing amniocentesis not possible in an intact pregnancy. Upper genital tract infection has four stages:

1. deciduitis

2. chorioamnionitis

3. amniotic fluid infection

4. infection of the fetus. Amniocentesis is regarded as the gold standard for diagnosing infection but will only be positive in stage 3 or 4 disease ${ }^{6}$

The possibility of either PTL or PPROM being the presenting symptom is explained by the following hypothesis ${ }^{7}$ : the onset of labour in the presence of infection (remote or local) is controlled by the host and regulation of different components of the host response determines the clinical presentation. If activation of the host response leads to secretion of uterotonic agents (prostaglandins) PTL will result. If on the other hand activation of the host response leads to the production of proteases (leucocyte elatase and matrix metalloproteinases) $\mathrm{PPROM}$ is more likely to result.

Infection is an important factor in the pathogenesis of PTL and PPROM but does not account for all cases and the condition should probably be considered a syndrome with 
multiple causes. Clinical chorioamnionitis is a condition that is easy to diagnose with recommended treatment protocols. The presumed diagnosis for non-infective or idiopathic PTL and PPROM is often considered sub-clinical chorioamnionitis. About half the cases of idiopathic preterm birth are associated with histological evidence of inflammation on histology. ${ }^{5}$ The 'inflammation' may not always be caused by bacterial infections causing confusion and perhaps explaining why treatment with antibiotics is not always successful.

There are a number of conditions that are associated with an increased risk for both PTL and PPROM:

\section{Table 1: Risk factors}

\begin{tabular}{|l|l|}
\hline PTL & PPROM \\
\hline Previous preterm birth & Infection \\
Low body mass & (STI, UTI) \\
Poor weight gain & Uterine distention \\
Heavy work load & Cervical incompetence \\
Uterine abnormalities & African-American \\
Psychological stress & Low socioeconomic status \\
Drug abuse (including smoking) & Drug abuse (including smoking) \\
Teenage pregnancy & 1st and/or 2nd trimester bleeding \\
\hline Abbreviations: STI, sexually transmitted infections; UTI, urinary tract \\
infection
\end{tabular}

With a baseline risk of $8 \%$, the risk of recurrent preterm birth after one, two and three consecutive preterm births are 15\%, 30\% and $45 \%$ respectively. ${ }^{8}$ These associations, particularly susceptibility to infections on a population basis, suggest a genomic relationship to preterm birth in an individual. Genetic polymorphisms are strongly linked to changes in the vaginal microflora and this may explain why some individuals and groups experience repeated preterm birth. No one polymorphism as been isolated and in all probability it will be a group of variations that will be responsible for the clinical presentation.

\section{Research results}

Because of the variables discussed above it is easy to appreciate why studies researching PTL and PPROM often produce inconsistent results. To try and address the problem Kenyon et $\mathrm{l}^{9,10}$ conducted the ORACLE trials. ORACLE I investigated PPROM and ORACLE II investigated PTL. Both were prospective randomized trials that compared various antibiotics (alone and in combination) with placebo. The results of ORACLE I showed that women on erythromycin (PPROM) experienced a range of health benefits for the neonate and thus a probable reduction in childhood disability. Co-amoxiclav was however associated with an increase of necrotizing enterocolitis in the neonate and should not be routinely recommended. A Cochrane Systematic Review on "Antibiotics for PROM"11 showed that the treatment was associated with a significant reduction in chorioamnionitis and prolongation of pregnancy beyond 48 hours and 7 days supporting the routine use of antibiotics in PPROM.

ORACLE II on the other hand showed no benefit to the neonate and the recommendation was that antibiotics should not routinely be prescribed to patients in PTL in the absence of infection. Antibiotic prescription was associated with lower levels of maternal infective complications.

There are a few concerns in extrapolating the ORACLE II data to different populations from those in the trial. One of the concerns was that the criteria for diagnosing PTL were probably inadequate as $63.5 \%$ of the patients enrolled delivered after 37 weeks gestation. Women with lower abdominal pain suggestive of labour pains, but closed cervixes were included. This also highlights the problem of accurately diagnosing PTL. The prevalence of infection as a cause of preterm labour in developed countries is given as around 15\%. Hence ORACLE II probably never had sufficient patients with infection in true preterm labour to detect any benefit of antibiotics in cases with intact membranes.

A similar Cochrane Review on "Prophylactic antibiotics for inhibiting preterm labour with intact membranes" ${ }^{12}$ failed to demonstrate a clear overall benefit on neonatal outcomes and the recommendation was that antibiotics should not be administered routinely.

The 7 year outcomes of children enrolled in the ORACLE trials have recently been published. ${ }^{13,14}$ The administration of erythromycin and/or co-amoxiclav in PTL was associated with a significantly increased risk of cerebral palsy in these children. When both antibiotics were given together the risk was greatest (35 of 769 , or $4.55 \%$ ), compared with erythromycin alone (18 of 785, 2.29\%) co-amoxyclav alone (15 of $763,1.97 \%$ ) or placebo alone (12 of $735,1.63 \%$ ). The short term gains from giving antibiotics to patients with PPROM have not been counterbalanced by any long term disadvantage in the children evaluated 7 years after treatment.

Comment in the Lancet editorial ${ }^{15}$ is clear: antibiotics are not risk free and we should guard against popular opinion "might as well give them, they don't do any harm". The reason for this is unclear but when sub-clinical infection is initiating labour, treatment with low-dose antibiotics might suppress rather than eradicate the infection. The association between perinatal infection and neurological damage is well described and allowing the pregnancy to continue might well predispose towards this situation. In cases of clinical chorioamnionitis the mother receive much higher doses of antibiotics intravenously. They further worryingly comment that in the UK erythromycin-resistant group-B streptococcus has risen from $6.4 \%$ in 2002 to $11.2 \%$ in 2006 . It is however unknown if treatment of PTL contributed to this rise.

\section{Local considerations}

One of the major problems experienced is trying to decide if trials conducted in developed world settings are applicable to South African conditions. A randomized multicentre South African trial (the PRAM study) comparing the use of ampicillin and metronidazole versus nothing found the group receiving nothing delivered at a median of 2 days whereas the ampicillin/metronidazole group delivered at a median of 14 days. There was also a significant reduction in neonatal morbidity in the ampicillin group. ${ }^{16}$ The trial was conducted in public hospitals around the country. As the ORACLE II trial was not powered to assess the role of antibiotics in cases with true preterm labour due to infections it might be appropriate in public hospitals to use the findings of the PRAM trial and in 
private patients (more likely to be similar to those in the ORACLE II trial) not to use antibiotics.

HIV infection is another confounder. Approximately 30\% of pregnant women attending public antenatal clinics are HIV infected. What is uncertain is the incidence of PTL in these patients and the role it plays in perinatal loss. It is also known that rupture of membranes for more than 4 hours is associated with an increase in Mother to Child Transmission of HIV. What is uncertain is whether there is greater benefit to the child by delaying delivery to give steroids or if the child does better being delivered immediately with the risks of prematurity and later HIV infection. Preliminary evidence from Chris Hani-Baragwanth Hospital indicates that the rate of infection in mothers that are HIV infected is not increased with expectant management (Personal communication, E Buchmann).

\section{Management suggestions}

The short term gains from giving antibiotics to patients with PPROM have not been counterbalanced by any long term disadvantage in the children evaluated 7 years after treatment and should be given. It would be prudent to avoid amoxyclav.

All HIV infected women and "3rd World patients" with PTL should be treated with erythromycin/ampicillin and metronidazole until there is evidence that the treatment is ineffective or causes harm. This treatment should probably not be given to "lst World patients". It is important to be aware of this new information in order to offer patients evidence-based treatment.

\section{References}

1. Pattinson RC, Velaphi S, Hardy B, Moran N, Steyn W. Overview. In Saving Babies 2006-2007: Sixth perinatal care survey of South Africa, Ed RC Pattinson. Tshepesa Press, Pretoria 2009, 1-65.

2. National Center for Health Statistics. Final 2002 natality data. Available at: www.marchofdimes.com/peristats.

3. Moutquin JM. Classification and heterogenicity of preterm birth. BJOG 2003;1 10 (suppl 20):30-3.

4. Newton ER. Preterm Labor, Preterm Premature Rupture of Membranes, and Chorioamnionitis. Clin Perinatol 32 (2005); 571 600.

5. Goncalves LK, Chaiworapongsa T, Romera R. Intrauterine infection and prematurity. Ment retard Dev Disabil Res 2002; 8: 3-13.

6. Romero R, Mazor M, Brandt F, et al:Interleukin lalpha and interleukin 1 beta in preterm nad term parturition. Ann NY Acad SCl 734:414-429, 1994.

7. Gomez R, Romero R, Edwin SS, David C. Pathogenesis of preterm labor and preterm rupture of membranes associated with intraamniotic infection. Infect Dis Clin N Am 11 (1): 135-166, 1997.

8. Bakketieg LS, Hoffman HJ. Epedemiology of preterm birth: results of a longitudinal study in Norway. In: Preterm labour. London: butterworths; 1981.

9. Kenyon SL, Taylor DJ, Tarno-Mordi W, for the ORACLE Collaborative Group. Broad-spectrum antibiotics for preterm, prelabour rupture of fetal membranes: the ORACLE I randomized trial. Lancet 2001; 357: 979-88.

10. Kenyon SL, Taylor DJ, Tarno-Mordi W, for the ORACLE Collaborative Group. Broad-spectrum antibiotics for spontaneous preterm labour the ORACLE II randomized trial. I I Kenyon S, Pike K, Jones DR, et al. Childhood outcomes after prescription of antibiotics to pregnant women with preterm rupture of membranes: 7 year follow-up of the ORACLE I trial. Lancet 2008; published online Sept 18. Lancet 2001; 357:989-94.

12. Kenyon S, Boulvain M, Nielson JP. Antibiotics for preterm rupture of membranes. Cochrane database of Systematic Reviews 2003, Issue 2. Art. No.:CD001058

13. King JF, Flenady V. Prophylactic antibiotics for inhibiting preterm labour with intact membranes. Cochrane Database of Systematic Reviews 2002, issue 4. Art. No.: CD00246.

14. Kenyon S, Pike K, Jones DR, et al. Childhood outcomes after prescription of antibiotics to pregnant women with preterm rupture of membranes: 7 year follow-up of the ORACLE I trial. Lancet 2008; published online Sept 18. DOI: 10.1016/S0140-6736(08)61202-7.

15. Kenyon S, Pike K, Jones DR, et al. Childhood outcomes after prescription of antibiotics to pregnant women with spontaneous preterm labour: 7 year follow-up of the ORACLE II trial. Lancet 2008; published online Sept 18. DOI:10.1016/S0140-6736(08)612038.

16. Bedford Russell AR, Steer PJ.Antibiotics in preterm labour - the ORACLE speaks. Lancet 2008; published online Sept 18: DOI:10.1016/S0140-6736(08)61248-9.

17. Norman K, Pattinson RC, De Sousa J, De Jong P, Moller G, Kirsten G. Ampicillin and metronidazole treatment in preterm labour: a multicentre, randomised controlled trial. Br J Obstet Gynaecol 1994; 101: 404-408 